MMCA-97 Conference, pp. 48-60

R. Čiegis (Ed)

(C) 1997 Vilniaus Gedimino technikos universitetas

\title{
ON UNIFORM IN TIME ERROR ESTIMATES FOR INVESTIGATION OF NONLINEAR DIFFERENCE SCHEMES
}

RAIMONDAS ČIEGIS and OLGA ŠTIKONIENE

Institute of Mathematics and Informatics

Akademijos 4, LT-2600 Vilnius, Lithuania

e-mail:rc@fm.vtu.lt

\section{INTRODUCTION}

One general methodology for investigation of difference schemes, which approximate nonstationary nonlinear differential equations, is given in [1,2]. That methodology is based on the nonlinear stability definition and enables us to use the investigation formula "aproximation + stability = convergence" in the nonlinear cases.

In this note we modify this scheme of investigation by using the definition of asymptotical stability of nonlinear difference schemes. It enables us to prove uniform in time error estimates. The efficiency of such methodology is demonstrated for implicit and explicit finite - difference schemes which approximate the semilinear diffusion-reaction problems

$$
\begin{array}{ll}
\frac{\partial u}{\partial t}=\sum_{j=1}^{d} \frac{\partial}{\partial x_{j}}\left(a_{j}(x) \frac{\partial u}{\partial x_{j}}\right)+f(x, t, u) & \text { in } \Omega \times(0, \infty), \\
u=0 & \text { on } \partial \Omega \times[0, \infty) \\
u(\cdot, 0)=u_{0} & \text { in } \Omega .
\end{array}
$$

Here $\Omega$ is a rectangular domain in $R^{d}, d \geq 1, a_{j}(x)$ are given functions satisfying conditions

$$
0<A_{L} \leq a_{j}(x) \leq A_{R} \quad \text { in } \quad \Omega .
$$

In this paper we use the following norm convention

$$
\|v(\cdot, t)\|_{L_{\infty}}=\sup _{x \in \Omega}|v(x, t)| .
$$


Then we define the neighbourhood of the solution

$$
B(u, R)=\left\{v:\|u(\cdot, t)-v(\cdot, t)\|_{L_{\infty}} \leq R\right\} .
$$

We assume that the differential equation has some structure which forces a solution to approach an equilibrium. More precisely, we assume the following:

(H1) $f(x, t, v) \underset{t \rightarrow \infty}{\longrightarrow} F(x, v)$.

(H2) The problem (1.1) - (1.3) defines asymptotically stable nonlinear operator in $B(u, R)$, i.e.:

$$
\lambda_{\max }(t)=\max _{\Phi \in H_{0}^{1}} \frac{\int_{\Omega}\left[-\sum_{j=1}^{d} a_{j}(x) \Phi_{x_{j}}^{2}+f_{u}^{\prime}(\cdot, t, U) \Phi^{2}\right] d x}{\int_{\Omega} \Phi^{2} d x}<0
$$

for any $U \in B(u, R)$, here $H_{0}^{1}$ is the subspace of the standard Sobolev space $H^{1}(\Omega)$ satisfying the homogeneous Dirichlet boundary conditions.

Then $\|u(\cdot, t)-\bar{U}\|_{L_{\infty}} \rightarrow 0$, where $\bar{U}$ satisfies the stationary problem

$$
\begin{array}{ccc}
-\sum_{j=1}^{d} \frac{\partial}{\partial x_{j}}\left(a_{j}(x) \frac{\partial \bar{U}}{\partial x_{j}}\right)=F(x, \bar{U}) & \text { in } \quad \Omega, \\
\bar{U}=0 & \text { on } \quad \partial \Omega .
\end{array}
$$

Following [2] (see, also [3]) in the difference scheme to be described below we replace function $f$ by a smooth function $f_{R}$, which coincides with $f$ in $B(u, R)$. Outside of $B(u, R) f_{R}$ is bounded and Lipschitz continuous, where all bounds depend only on $u$ and $R$. This replacement does not affect the exact solution $u(x, t)$.

Finally we mention some related work. Larsson [3] analyses the long-time behavior of the dissipative backward Euler method. He proves error estimates in the $L_{2}$ norm. The approximation of "contracting" trajectories near asymptotically stable equilibra by an explicit Euler finite-difference scheme is considered by Sanz-Serna and Stuart [4]. The qualitative behaviour of spatially semidiscrete finite element solutions of a semilinear parabolic problem near an unstable hyperbolic equilibrium is studied by Larsson and Sanz-Serna [5]. We mention the important work of Heywood and Rannacher [6].

\section{FINITE-DIFFERENCE SCHEME}

In this section we describe the finite-difference approximation of (1.1) - (1.3).

Let $\Omega_{\tau}$ be the uniform time mesh with the time step $\tau$. Let $\Omega_{h}=\Omega_{1 h} \times$ $\Omega_{2 h} \times \cdots \times \Omega_{d h}$ be a discretization of $\Omega$, where $\Omega_{i h}$ are space meshes obtained 
by dividing space intervals ] $0,1[$ into mesh intervals by a sequence of points $x_{i j}=j h, j=1,2, \ldots, N-1$, where $N h=1$ and $h$ denotes the space step. Then for any vector $j=\left(j_{1}, j_{2}, \ldots, j_{d}\right)$ with $0<j_{k}<N$ we get a discrete point $X_{j}=\left(x_{1 j_{1}}, \ldots, x_{d_{j_{\alpha}}}\right) \in \Omega_{h}$.

Let $U_{j}^{n}$ denotes the discrete approximation of $u\left(X_{j}, t_{n}\right)$. We also use the notation

$$
V(x+\alpha ; k)=V\left(x_{1}, \ldots, x_{k}+\alpha, \ldots, x_{d}\right) .
$$

The inner product between mesh functions $U$ and $V$ and the discrete $L_{2}$ and $L_{\infty}$ norms are defined by

$$
\begin{aligned}
& (U, V)=h^{d} \sum_{X \in \Omega_{h}} U(X) V(X), \quad\|V\|=\sqrt{(V, V)}, \\
& \|V\|_{\infty}=\max _{X \in \Omega_{h}}|V(X)| .
\end{aligned}
$$

The finite-difference scheme is defined as follows

$$
\begin{aligned}
& \frac{U^{n+1}-U^{n}}{\tau}=\sum_{k=1}^{d} D_{k} U^{\sigma}+f_{R}\left(X, t_{n+\sigma}, U^{\sigma}\right) \quad \text { in } \Omega_{h}, \\
& U^{n+1}=0 \quad \text { on } \partial \Omega_{h}, \\
& U(X, 0)=u_{0}(X) \quad \text { in } \Omega_{h} \cup \partial \Omega_{h},
\end{aligned}
$$

where $D_{k}$ is an approximation of the differential operator by central differences

$$
\begin{aligned}
D_{k} U= & \frac{1}{h^{2}}\left[a_{k}\left(X+\frac{h}{2} ; k\right)(U(X+h ; k)-U(X))\right. \\
& \left.-a_{k}\left(X-\frac{h}{2} ; k\right)(U(X)-U(X-h ; k))\right],
\end{aligned}
$$

and we use the following notations

$$
U^{\sigma}=\sigma U^{n+1}+(1-\sigma) U^{n}, \quad t_{n+\sigma}=t_{n}+\sigma \tau, \quad 0 \leq \sigma \leq 1 .
$$

It is well-known that $D_{k}$ satisfies the estimates (see, e.g. [6])

$$
\frac{4 A_{L}}{h^{2}} \sin ^{2}\left(\frac{\pi h}{2}\right)\|U\|^{2} \leq\left(-D_{k} U, U\right) \leq \frac{4}{h^{2}} A_{R}\|U\|^{2}
$$

\section{THE METHOD FOR INVESTIGATION OF NONLINEAR DIFFERENCE SCHEMES}

In this section we consider a modification of a general investigation method which was proposed in $[1,2]$. 
First the existence of the unique solution of finite-difference scheme (2.1) (2.3) is proved. We find a solution by using the iterative method

$$
\begin{array}{ll}
\frac{\stackrel{s+1}{U}-U^{n}}{\tau}=\sum_{k=1}^{d} D_{k} \stackrel{s+1_{\sigma}}{U}+f_{R}\left(X, t_{n+\sigma}, \stackrel{s}{U^{\sigma}}\right) & \text { in } \Omega_{h}, \\
\stackrel{s+1}{U}=0 & \text { on } \partial \Omega_{h}, \\
\stackrel{\circ}{U}=U^{n}, &
\end{array}
$$

where $\stackrel{s}{U}$ denotes the $s$-th iterative approximation and $\stackrel{s}{U^{\sigma}}=\sigma \stackrel{s}{U}+(1-\sigma) U^{n}$.

As it follows from [2] the convergence of the iterative method (3.1) - (3.2) and the uniqueness of the solution depends on the following stability property.

Let us consider the auxiliary discrete problem for the difference $V^{n+1}-$ $W^{n+1}$

$$
\begin{aligned}
& \frac{V^{n+1}-W^{n+1}}{\tau}=\sigma \sum_{k=1}^{d} D_{k}\left(V^{n+1}-W^{n+1}\right) \\
& +f_{R}\left(X, t_{n+\sigma}, \sigma P^{n+1}+(1-\sigma) U^{n}\right) \\
& -f_{R}\left(X, t_{n+\sigma}, \sigma Q^{n+1}+(1-\sigma) U^{n}\right) \text { in } \Omega_{h}, \\
& V^{n+1}-W^{n+1}=0 \quad \text { on } \partial \Omega_{h},
\end{aligned}
$$

where $U^{n} \in B\left(u\left(t_{n}\right), R\right)$ and $P^{n+1}, Q^{n+1}$ are any functions satisfying boundary conditions (3.4).

DEFINITION 1. The finite-difference scheme is said to be stable, if for sufficiently small $\tau \leq \tau_{0}, h \leq h_{0}$ the following estimate

$$
\left\|V^{n+1}-W^{n+1}\right\|_{(1)} \leq \tau C_{D}\left\|P^{n+1}-Q^{n+1}\right\|_{(1)}
$$

holds for problem (3.3) - (3.4), where $C_{D}$ may depend on constants which are used for the definition of $f_{R}$.

Then the following theorem is proved in [2].

THEOREM 3.1. If the difference scheme $(2.1)-(2.3)$ is stable then for sufficiently small $\tau \leq \tau_{1}$ the iterative sequence defined by problem $(3.1)-(3.2)$ converges to the solution of the difference scheme (2.1) - (2.3), the following estimate

$$
\left\|U^{n+1}-\stackrel{s}{U}\right\|_{(1)} \leq \frac{q^{s}}{1-q}\left\|\stackrel{1}{U}-U^{n}\right\|_{(1)}, \quad q<1
$$

holds and this solution is unique.

Now we will give one important remark about the realization details of the iterative method (3.1) - (3.2). 
REMARK 1. The main norm $\|\cdot\|_{(1)}$ can be weaker than the maximum norm $\|\cdot\|_{\infty}$. We will prove below that for sufficiently small $\tau \leq \tau_{0}$ the solution $U^{n+1} \in B\left(u\left(t_{n+1}\right), R\right)$. Hence we have that $f_{R}\left(X, t_{n+\sigma}, U^{\sigma}\right)=$ $f\left(X, t_{n+\sigma}, U^{\sigma}\right)$. In the formulation of the iterative method we can use a ball $B\left(U^{n}, 2 R\right)$. If after convergence of the iterative sequence $U^{n+1} \notin B\left(U^{n}, 2 R\right)$, we decrease the time step $\tau$. It is important to note that some $\stackrel{s}{U}$ may not belong to $B\left(U^{n}, 2 R\right)$ (nor to $\left.B\left(u\left(t_{n+1}\right), R\right)\right)$.

Now we will investigate the convergence of the discrete solution. The global error $Z_{j}^{n}=U_{j}^{n}-u\left(X_{j}, t_{n}\right)$ satisfies the problem

$$
\begin{array}{ll}
\frac{Z^{n+1}-Z^{n}}{\tau}=\sum_{k=1}^{d} D_{k} Z^{\sigma}+f_{R}\left(X, t_{n+\sigma}, U^{\sigma}\right) \\
& -f_{R}\left(X, t_{n+\sigma}, u^{\sigma}\right)+\Psi^{n} \quad \text { in } \Omega_{h}, \\
Z^{n}=0 & \text { on } \partial \Omega_{h}, \\
Z(X, 0)=0 & \text { in } \Omega_{h} \cup \partial \Omega_{h},
\end{array}
$$

where the function

$$
\Psi_{j}^{n}=-\frac{u^{n+1}-u^{n}}{\tau}+\sum_{k=1}^{d} D_{k} u^{\sigma}+f\left(x, t_{n+\sigma}, u^{\sigma}\right)
$$

is called the truncation error.

DEFINITION 2. Finite-difference scheme (2.1)-(2.3) is said to be asymptotically stable, if for sufficiently small $\tau \leq \tau_{0}, h \leq h_{0}$ the following estimate

$$
\left\|Z^{n+1}\right\|_{(1)} \leq e^{-C_{S} \tau}\left\|Z^{n}\right\|_{(1)}+\tau C_{T}\left\|\Psi^{n}\right\|_{(2)},
$$

holds for problem (3.6) - (3.7), where $C_{S}, C_{T}$ are nonnegative constants that may depend on constants used in the definition of function $f_{R}$.

Let assume that the following estimate

$$
\left\|\Psi^{n}\right\|_{(2)} \leq C_{A}\left(\tau^{\alpha}+h^{\beta}\right), \quad \alpha, \beta>0, n \geq 0
$$

is valid for the truncation error.

THEOREM 3.2. Let finite-difference scheme (2.1)-(2.3) be asymptotically stable. Then for sufficiently small $\tau \leq \tau_{3}$ the global error of the solution of difference scheme (2.1) - (2.3) satisfies the uniform in time estimate

$$
\left\|U^{n}-u\left(t_{n}\right)\right\|_{(1)} \leq C\left(\tau^{\alpha}+h^{\beta}\right) .
$$


P r o o f. By iterating the stability estimate (3.8) we have

$$
\begin{aligned}
\left\|Z^{n}\right\|_{(1)} \leq e^{-C_{S}\left(t_{n}-t_{m}\right)}\left\|Z^{n-m}\right\|_{(1)} & \\
& +\tau C_{T} \max _{n-m \leq j \leq n-1}\left\|\Psi^{j}\right\|_{(2)}\left(1+e^{-C_{S} \tau}+\cdots+e^{-C_{S}(m-1) \tau}\right) .
\end{aligned}
$$

With $\tau \leq \tau_{0}$ we thus get

$$
\left\|Z^{n}\right\|_{(1)} \leq e^{-C_{S}\left(t_{n}-t_{m}\right)}\left\|Z^{n-m}\right\|_{(1)}+\frac{C_{T}}{C_{S}} e^{C_{S} \tau_{0}} \max _{n-m \leq j \leq n-1}\left\|\Psi^{j}\right\|_{(2)} .
$$

Taking $t_{m}=t_{0}$ and using (3.8) we prove the required uniform in time global error estimate (3.10). This completes the proof.

There we will make one important remark (see also [2,3]). Although the replacement of $f(x, t, u)$ by $f_{R}(x, t, u)$ does not affect the exact solution of the differential problem, it may change the solution of the difference scheme. Hence it is necessary additionally to prove the convergence estimate in the maximum norm $L_{\infty}$. Examples of such analysis will be given below.

Next we consider the corresponding discrete stationary problem

$$
\begin{array}{ll}
-\sum_{k=1}^{d} D_{k} V=F_{R}(x, V) & \text { in } \quad \Omega_{h}, \\
V=0 & \text { on } \quad \partial \Omega_{h} .
\end{array}
$$

The existence of the solution $V$ is guaranted for small $h$ if $F_{R}$ satisfies the assumption (H2) and since $V$ is a finite-dimensional vector.

Then finite-difference scheme (2.1)-(2.3) can be used as an iterative method for finding the solution of stationary problem (3.12).

THEOREM 3.3. Let finite-difference scheme (2.1)-(2.3) is asymptotically stable. Then $U^{n}$ converges to the stationary solution $V$ of (3.12) and the following error estimate is valid

$$
\left\|U^{n}-V\right\|_{(1)} \leq e^{-C_{S} t_{n}}\left\|U^{0}-V\right\|_{(1)} .
$$

Moreover, $V$ converges to a stationary solution $\bar{U}$ of (1.5) and $V-\bar{U}$ satisfies the estimates

$$
\|V-\bar{U}\|_{(1)} \leq C h^{\beta} .
$$

P r o o f. The difference $Z^{n}=U^{n}-V$ satisfies the problem

$$
\frac{Z^{n+1}-Z^{n}}{\tau}=\sum_{k=1}^{d} D_{k} Z^{\sigma} .
$$


Then it follows from (3.11) that

$$
\left\|Z^{n}\right\| \leq e^{-C t_{n}}\left\|Z^{0}\right\| .
$$

Hence an initial error $\left\|Z^{0}\right\|$ is reduced $1 / \varepsilon$ times if $n \geq \ln (1 / \varepsilon) /\left(C_{S} \tau\right)$.

We now turn to the proof of (3.14). Substituting $\bar{U}$ into (2.1) we get that the truncation error satisfies

$$
\|\Psi\|_{(2)} \leq C_{A} h^{\beta}, \quad \beta>0 .
$$

Then by (3.11) we obtain the uniform in time error estimate

$$
\left\|U^{n}-\bar{U}\right\| \leq e^{-C_{S} t_{n}}\left\|U^{0}-\bar{U}\right\|+\frac{C_{T}}{C_{S}} h^{\beta} .
$$

Using this inequality and the established fact that $U^{n} \rightarrow V$ as $n \rightarrow \infty$ we prove (3.14). The theorem is proved .

\section{THE IMPLICIT FINITE-DIFFERENCE SCHEME}

In this section we apply general results of Section 3 to the implicit scheme $(2.1)-(2.3)$ with $\sigma=1$

$$
\begin{array}{lr}
\frac{U^{n+1}-U^{n}}{\tau}=\sum_{k=1}^{d} D_{k} U^{n+1}+f_{R}\left(X, t_{n+1}, U^{n+1}\right) & \text { in } \Omega_{h}, \\
U^{n+1}=0 & \text { on } \partial \Omega_{h} .
\end{array}
$$

We assume the following hypotheses.

(H3) The function $f_{R}$ is globally Lipschitz function, i.e.

$$
\left|f_{R}\left(x, t, U_{1}\right)-f_{R}\left(x, t, U_{2}\right)\right| \leq L\left|U_{1}-U_{2}\right| .
$$

(H4) The smooth function $f_{R}$ satisfies the estimate

$$
\frac{\partial f_{R}}{\partial u}(x, t, v) \leq-S_{F}, \quad S_{F}>0 .
$$

It is well-known that for a sufficiently smooth solution $u(x, t)$ of $(1.1)-$ (1.3) the truncation error satisfies

$$
|\Psi| \leq C_{A}\left(\tau+h^{2}\right) \quad \text { in } \quad \Omega_{h} .
$$


It is sufficient to prove that finite-difference scheme (4.1)-(4.2) is stable and asymptotically stable.

LEMMA 4.1. Let assume that (H3) and (H4) are satisfied. Then finitedifference scheme (4.1) is stable and asymptotically stable in $L_{\infty}$, i.e. $\|\cdot\|_{(j)}=$ $\|\cdot\|_{\infty}, j=1,2$.

$\mathrm{P}$ r o o f. In order to prove that scheme (4.1) is stable we consider the auxiliary discrete problem (see (3.5))

$$
\begin{aligned}
\frac{V^{n+1}-W^{n+1}}{\tau}= & \sum_{\substack{k=1\\
}}^{\infty} D_{k}\left(V^{n+1}-W^{n+1}\right)+f_{R}\left(X, t_{n+1}, P^{n+1}\right) \\
& -f_{R}\left(X, t_{n+1}, Q^{n+1}\right)
\end{aligned}
$$

It follows from the maximum principle that

$$
\left\|V^{n+1}-W^{n+1}\right\|_{\infty} \leq \tau\left\|f_{R}\left(\cdot, t_{n+1}, P^{n+1}\right)-f_{R}\left(\cdot, t_{n+1}, Q^{n+1}\right)\right\|_{\infty} .
$$

By the estimate (H3) we now have that for $n \geq 0$

$$
\left\|V^{n+1}-W^{n+1}\right\|_{\infty} \leq \tau L\left\|P^{n+1}-Q^{n+1}\right\|_{\infty} .
$$

We conclude that finite-difference scheme (4.1) - (4.2) is stable.

We now turn to the proof of asymptotical stability. The global error $Z=$ $U-u$ satisfies the discrete problem

$$
\begin{aligned}
& \frac{Z^{n+1}-Z^{n}}{\tau}=\sum_{k=1}^{d} D_{k} Z^{n+1}+f_{R}\left(X, t_{n+1}, U^{n+1}\right) \\
& -f_{R}\left(X, t_{n+1}, u\left(t_{n+1}\right)\right)+\Psi^{n} \text {. }
\end{aligned}
$$

Then it follows from (H4) and from the maximum principle that

$$
\left\|Z^{n+1}\right\|_{\infty} \leq \frac{1}{1+\tau S_{F}}\left(\left\|Z^{n}\right\|_{\infty}+\tau\left\|\Psi^{n}\right\|_{\infty}\right) .
$$

We note that

$$
\frac{1}{1+\tau S_{F}}=e^{-\tau C_{F}} \quad \text { for } \quad C_{F}>0 .
$$

This completes the proof of the lemma.

Hence the conclusions of Theorem 3.1 and Theorem 3.2 hold for the implicit difference scheme $(4.1)-(4.2)$ if the function $f_{R}$ satisfies assumptions $(\mathrm{H} 3)$ and $(\mathrm{H} 4)$.

We also remark that, since the asymptotical stability is proved in the maximum norm $L_{\infty}$, we have

$$
\left\|U^{n}-u\left(t_{n}\right)\right\|_{\infty} \leq R, \quad 0 \leq t_{n}<\infty
$$


for $\tau$ and $h$ sufficiently small, so that, in fact,

$$
f_{R}\left(X, t_{n}, U^{n}\right)=f\left(X, t_{n}, U^{n}\right) .
$$

Now we replace the assumptions (H3), (H4) with the following more general assumption:

(H5) The smooth function $f_{R}$ satisfies globally the estimates

$$
-S_{L} \leq \frac{\partial f_{R}(x, t, v)}{\partial u} \leq S_{R} \quad \text { for } \quad S_{L}, S_{R}>0 .
$$

In order to use the results of Section 3 we will prove the stability estimates in Lemma 4.2 and Lemma 4.3.

LEMMA 4.2. Let $f_{R}$ be a smooth function satisfying (H5). Then finitedifference scheme (4.1) is stable in $L_{\infty}$.

$\mathrm{P} \mathrm{r} \mathrm{o} \mathrm{o} \mathrm{f.} \mathrm{It} \mathrm{is} \mathrm{sufficient} \mathrm{to} \mathrm{note} \mathrm{that} \mathrm{the} \mathrm{assumption} \mathrm{(H3)} \mathrm{follows} \mathrm{from} \mathrm{the}$ assumption (H5) with $L=\max \left(S_{L}, S_{R}\right)$. Then the stability inequality (3.5) follows from Lemma 4.1. This completes the proof of Lemma 4.2.

LEMMA 4.3. Let $f_{R}$ be a smooth function satisfying (H5) and the following inequality

$$
d A_{L} \lambda_{1}-S_{R}>C_{f}, \quad \lambda_{1}=\frac{4}{h^{2}} \sin ^{2}\left(\frac{\pi h}{2}\right), C_{f}>0
$$

is valid. Then finite-difference scheme (4.1) is asymptotically stable in $L_{2}$, i.e. the stability inequality

$$
\left\|Z^{n+1}\right\| \leq e^{-\tau C_{F}}\left(\left\|Z^{n}\right\|+\tau\left\|\Psi^{n}\right\|\right) \quad \text { for } \quad C_{F}>0
$$

holds for the global error.

P r o o f. We first split $f_{R}$ as $f_{R}=f_{R}^{+}-f_{R}^{-}$, where

$$
0 \leq \frac{\partial f_{R}^{+}}{\partial u} \leq S_{R}, \quad 0 \leq \frac{\partial f_{R}^{-}}{\partial u} \leq S_{L}
$$

Then we have the discrete problem for the global error

$$
\frac{Z^{n+1}-Z^{n}}{\tau}+\frac{\partial f_{R}^{-}}{\partial u} Z^{n+1}=\sum_{j=1}^{d} D_{j} Z^{n+1}+\frac{\partial f_{R}^{+}}{\partial u} Z^{n+1}+\Psi^{n} .
$$

Taking the inner product of (4.7) with $Z^{n+1}$ and using (4.6), we get

$$
\left\|Z^{n+1}\right\|^{2} \leq\left\|Z^{n}\right\|\left\|Z^{n+1}\right\|+\tau \sum_{j=1}^{d}\left(D_{j} Z^{n+1}, Z^{n+1}\right)
$$




$$
+\tau S_{R}\left\|Z^{n+1}\right\|^{2}+\tau\left\|\Psi^{n}\right\|\left\|Z^{n+1}\right\|
$$

Using (2.4) and (4.6), we have

$$
\left(1+\tau\left(d A_{L} \lambda_{1}-S_{R}\right)\right)\left\|Z^{n+1}\right\| \leq\left\|Z^{n}\right\|+\tau\left\|\Psi^{n}\right\|
$$

We note that (4.4) implies

$$
1+\tau\left(d A_{L} \lambda_{1}-S_{R}\right)=e^{\tau C_{F}} \quad \text { for } \quad C_{F}>0
$$

hence (4.5) follows from (4.8). This completes the proof of Lemma 4.3.

Since the asymptotical stability is proved only in the $L_{2}$ norm, we must prove that $U^{n} \in B\left(u\left(t_{n}\right), R\right)$. It follows from (4.3) and the well-known inverse inequality

$$
\left\|Z^{n}\right\|_{\infty} \leq h^{-d / 2}\left\|Z^{n}\right\|
$$

that the global error satisfies the estimate

$$
\left\|Z^{n}\right\|_{\infty} \leq C h^{-d / 2}\left(\tau+h^{2}\right)
$$

Hence, for $d \leq 3$ and $\tau=C_{0} h^{d / 2+\varepsilon}$ with $\varepsilon>0$, we have

$$
\left\|Z^{n}\right\|_{\infty} \leq C\left(h^{\varepsilon}+h^{2-d / 2}\right) \leq R
$$

for sufficiently small $h$ and $\tau$.

In the one dimensional case $d=1$ we can prove that $U^{n} \in B\left(u\left(t_{n}\right), R\right)$ without imposing the restrictive relation between sizes of parameters $\tau$ and $h$.

LEMMA 4.4. Let $d=1$ and $f_{R}$ be a smooth function satisfying $(H 5)$ and the following inequalities

$$
\left\|\frac{\partial f_{R}}{\partial x}\right\|_{\infty} \leq S_{F}, \quad A_{L} \lambda_{1}-S_{R}=C_{F}>0
$$

Then for sufficiently small $\tau$ and $h$ we have that $U^{n} \in B\left(u\left(t_{n}\right), R\right)$.

$\mathrm{P}$ r o o f. We will use the well-known multiplicative inequality (see, e.g. $[7])$

$$
\left.\left\|Z^{n}\right\|_{\infty} \leq C \| Z_{\bar{x}}^{n}\right]\left.\right|^{0.5}\left\|Z^{n}\right\|^{0.5}
$$

where we denote

$$
\left.\| Z_{\bar{x}}^{n}\right]\left.\right|^{2}=\sum_{j=1}^{N} h a\left(X_{j-0.5}\right)\left(\frac{Z_{j}-Z_{j-1}}{h}\right)^{2} .
$$


It is easy to see that

$$
\left.\left.\left.\| Z_{\bar{x}}^{n}\right] \mid \leq \| U_{\bar{x}}^{n}\right] \mid+\| u_{\bar{x}}^{n}\right] \mid .
$$

Since $u(x, t)$ is a smooth function, we have $\left.\| u_{\bar{x}}^{n}\right] \mid \leq C$. It remains to prove that $\left.\| U_{\bar{x}}^{n}\right] \mid$ is also globally bounded.

Taking the inner product of (4.1) with $D_{1} U^{n+1}$ and using the Green's first formula we get

$$
\begin{aligned}
\left.\| U_{\bar{x}}^{n+1}\right]\left.\right|^{2}+\tau\left\|D_{1} U^{n+1}\right\|^{2} \leq & \left.\left.\| U_{\bar{x}}^{n}\right] \mid \| U_{\bar{x}}^{n+1}\right] \mid \\
& \left.\left.+\tau\left(A_{R}\left\|\frac{\partial f_{R}}{\partial x}\right\|_{\infty}+S_{R} \| U_{\bar{x}}^{n+1}\right] \mid\right) \| U_{\bar{x}}^{n+1}\right] \mid .
\end{aligned}
$$

Using the inequality (see, e.g. [6])

$$
\left.\left\|D_{1} U^{n+1}\right\|^{2} \geq \lambda_{1} A_{L} \| U_{\bar{x}}^{n+1}\right]\left.\right|^{2}
$$

and the assumption (4.9), we get

$$
\left.\left.\left(1+\tau\left(\lambda_{1} A_{L}-S_{R}\right)\right) \| U_{\bar{x}}^{n+1}\right] \mid \leq \| U_{\bar{x}}^{n}\right] \mid+\tau A_{R} S_{F} .
$$

By iterating this inequality, we have the uniform estimate

$$
\left.\left.\| U_{\bar{x}}^{n}\right] \mid \leq \| U_{\bar{x}}^{0}\right] \mid+\left(1+\tau C_{F}\right) A_{R} S_{F} / C_{F} .
$$

Hence the global error $Z^{n}$ can be estimated in the $L_{\infty}$ norm by

$$
\left\|Z^{n}\right\|_{\infty} \leq C\left(\tau+h^{2}\right)^{0.5}
$$

and $U^{n} \in B\left(u\left(t_{n}\right), R\right)$ for sufficiently small $\tau$ and $h$. This completes the proof of Lemma 4.4.

\section{THE EXPLICIT FINITE-DIFFERENCE SCHEME}

In this section we apply general results of Section 3 to the explicit scheme $(2.1)-(2.3)$ with $\sigma=0$

$$
\begin{array}{ll}
\frac{U^{n+1}-U^{n}}{\tau}=\sum_{k=1}^{d} D_{k} U^{n}+f_{R}\left(X, t_{n}, U^{n}\right) & \text { in } \Omega_{h}, \\
U^{n+1}=0 & \text { on } \partial \Omega_{h} .
\end{array}
$$

Since we are using an explicit scheme, only asymptotical stability must be investigated. We again note that for a sufficiently smooth solution $u(x, t)$ of (1.1) - (1.3) the truncation error satisfies (4.3). 
Lemma 5.1. Let $f_{R}$ be a smooth function satisfying (H5) and the inequality (4.4) is valid. Then for sufficiently small $\tau \leq \tau_{0}$, where

$$
\tau_{0}=\frac{2}{A_{R} 4 d / h^{2}+S_{L}+C_{f}}
$$

the finite-difference scheme (5.1) - (5.2) is asymptotically stable.

$\mathrm{P}$ r o o f. The global error $Z=U-u$ satisfies the discrete problem

$$
\begin{aligned}
\frac{Z^{n+1}-Z^{n}}{\tau}= & \sum_{k=1}^{d} D_{k} Z^{n}+f_{R}\left(X, t_{n}, U^{n}\right) \\
& -f_{R}\left(X, t_{n}, u /\left(t_{n}\right)\right)+\Psi^{n}
\end{aligned}
$$

In our stability analysis we will use the method similar to one presented in [4]. Let define the following matrices

$$
A Z=\sum_{k=1}^{d} D_{k} Z, \quad T^{ \pm} Z=\frac{\partial f_{R}^{ \pm}}{\partial u} Z .
$$

Taking $L_{2}$ norms of (5.4), we obtain

$$
\left\|Z^{n+1}\right\| \leq\left\|I+\tau\left(A+T^{+}-T^{-}\right)\right\|\left\|Z^{n}\right\|+\tau\left\|\Psi^{n}\right\| .
$$

Since $A$ is symmetric and $T^{ \pm}$are diagonal the norm $\left\|I+\tau\left(A+T^{+}-T^{-}\right)\right\|$ coincides with the spectral radius $\rho$ of $I+\tau\left(A+T^{+}-T^{-}\right)$. Using (H5) and standard results on eigenvalues of the matrix $A$, we get

$$
\rho=\max \left(\left|1-\tau\left(A_{R} \frac{4 d}{h^{2}}+S_{L}\right)\right|,\left|1-\tau\left(A_{L} \lambda_{1}-S_{R}\right)\right|\right) .
$$

Taking $\tau \leq \tau_{0}$, where $\tau_{0}$ is given by (5.3), we get that $\rho=1-\tau C_{f}<$ $\exp \left(-\tau C_{f}\right)$. Hence it follows from (5.5) that

$$
\left\|Z^{n+1}\right\| \leq e^{-\tau C_{f}}\left\|Z^{n}\right\|+\tau\left\|\Psi^{n}\right\|
$$

This completes the proof of Lemma 5.1.

Again we must prove that $U^{n} \in B\left(u\left(t_{n}\right), R\right)$. Since $\tau \leq C h^{2}$ by (5.3), the required global error estimate follows for $d \leq 3$ and small $h$ from the inverse inequality

$$
\left\|Z^{n}\right\|_{\infty} \leq h^{-d / 2}\left\|Z^{n}\right\| \leq C h^{2-d / 2} .
$$




\section{REFERENCES}

[1] Raim. Čiegis, Rem. Čiegis and M. Meilūnas, On one general method for the investigation of difference schemes, Lith. Math. J., 36 (1996), $224-241$.

[2] Raim. Čiegis, Rem. Čiegis and O. Štikonienè, On the extension of one method for investigation of nonlinear difference schemes, Lith. Math. J., 37 (1997), $000-000$.

[3] S. Larsson, The long-time behavior of finite-element approximations of solutions to semilinear parabolic problems, SIAM J. Numer. Anal., 26 (1989), 348 - 365.

[4] J.M. Sanz-Serna, A.M. Stuart, A note on uniform in time error estimates for approximations to reaction-diffusion equations, IMA Journal of Numer. Anal., 12 (1992), $457-462$.

[5] S. Larsson and J.M. Sanz-Serna, The behavior of finite element solutions of semilinear parabolic problems near stationary points, SIAM J. Numer. Anal., 31 (1994), 1000 - 1018.

[6] A.A. Samarskij, The theory of difference schemes, Nauka, Moscow, 1986.

[7] O.L. Ladyzhenskaya, Boundary value problems of mathematical physics, Nauka, Moscow, 1973. 